\title{
ITK Inhibitor CPI-818
}

National Cancer Institute

\section{Source}

National Cancer Institute. ITK Inhibitor CPI-818. NCI Thesaurus. Code C162010.

An orally available, small-molecule, irreversible inhibitor of interleukin- 2 inducible T-cell kinase (ITK) with potential immunomodulatory and antineoplastic activities. Upon oral administration, ITK inhibitor CPI-818 selectively and covalently binds to the cysteine residue at position 442 (CYS-442) of ITK, thereby disrupting ITK-mediated signal transduction, while sparing tyrosine-protein kinase TXK (resting lymphocyte kinase, RLK) activity. This may abrog ate T-cell receptor (TCR) signaling through ITK and inhibit TCRinduced proliferation of malignant T-cells. Additionally, inhibiting ITK activation may prevent the upregulation of GATA-3, a transcription factor that drives T-helper 2 (Th2) cell differentiation and is overexpressed in certain T-cell lymphomas. Thus, selective inhibition of ITK may inhibit Th2 responses without affecting T-helper 1 (Th1)-dependent immunity. ITK, a member of the Tec family of non-receptor protein tyrosine kinases plays a significant role in the T-cell development, differentiation and production of proinflammatory cytokines. 